\title{
Prevalencia de enteroparásitos zoonóticos en perros (canis familiaris) y el nivel de cultura ambiental orientado a mascotas en Huánuco.
}

\author{
Prevalence of zoonotic intestinal parasites in dogs (canis familiaris) and level of pets-oriented \\ environmental culture in Huánuco.
}

Edward Huerto-Medina ${ }^{1}$, Abner Fonseca-Livias ${ }^{2}$, Bernardo Dámaso-Mata ${ }^{3}$

\section{RESUMEN}

Objetivo: Determinar la relación de la prevalencia de enteroparásitos zoonóticos en perros y el nivel de cultura ambiental orientado a mascotas en el Centro Poblado La Esperanza, Huánuco. Materiales y Métodos: Se realizó un estudio transversal-correlacional en diciembre del 2014, en 104 perros con dueño, de ambos sexos, diferentes edades y razas seleccionados por un muestreo bietápico. Se evaluaron dos muestras de heces por animal mediante el Método de Graban y de concentración (Método de flotación de Sheather y Método de Sedimentación simple en copas). Se definió como caso a los animales que resultaron positivos a helmintos al examen coproparasitológico. Resultados: La Prevalencia total por uno o más helmintos fue de $92,3 \%$, el parásito hallado con mayor frecuencia fue Ancylostoma caninum 72,1\%, seguido de Toxocara canis 54,8\%, Taenia sp. 20,2\%, Toxascaris leonina 19,2\% y Dipylidium caninum 13,5\%. Según el nivel de cultura ambiental orientado a mascotas, de los 104 dueños encuestados; el 7,7\% posee el nivel bajo, el 34,6\% poseen el nivel medio y el $57,7 \%$ poseen el nivel alto. No existe relación significativa (ya que se obtuvo un valor rho $=-0,044$, con un $\mathrm{P}$ sig. $=0,658)$ entre la prevalencia de enteroparásitos zoonóticos en perros y el nivel de cultura ambiental orientado a mascotas. Conclusion: La prevalencia encontrada de estas parasitosis representa un gran riesgo para la salud tanto animal como humana, por lo tanto, se hace necesario implementar acciones de educación sanitaria a la comunidad y la elaboración de planes de desparasitación para las mascotas.

Palabras Clave: Prevalencia, enteroparásitos zoonóticos, Canis familiaris, cultura ambiental orientado a mascotas.

\begin{abstract}
Objective: To determine the relationship of the prevalence of zoonotic intestinal parasites in dogs and level of pets-oriented environmental culture at minor town, La Esperanza Huánuco. Materials and Methods: A transverse correlational study was conducted in December 2014. We collected 104 owned dogs, both sexes, different ages and races. Sample was obtained by two-stage sampling. Two stool samples per animal were evaluated by Graban and concentration methods. Two samples per animal were evaluated by the method of Record and concentration (Sheather's flotation and Sedimentation methods in cups). The animals that tested positive for helminths to copro parasitological examination were defined as a case. Results: The overall prevalence of one or more helminth was $92,3 \%$. The most common parasite was Ancylostoma caninum $(72,1 \%)$. The next parasites were Toxocara canis $(54,8 \%)$, Taenia sp. $(20,2 \%)$, Toxascaris leonine $(19,2 \%)$ and Dipylidium caninum (13,5\%). The level of pets-oriented environmental culture of owners (104 respondents) were low level $(7,7)$, medium level $(34,6 \%)$ and highest level $(57,7 \%)$. There is no statistical significance (rho $=-0,044 ; \mathrm{P}=0,658$ ) between the prevalence of zoonotic intestinal parasites in dogs and the level of pets-oriented environmental culture. Conclusion: The prevalence of these parasites is a major health risk for both animal and human; therefore it is necessary to implement actions of health education to the community and drawing up plans for deworming pets.
\end{abstract}

Key words: prevalence, Intestinal Diseases, Parasitic, Zoonoses, Canis familiaris, Environmental Education.

\footnotetext{
Médico Veterinario. Dr. Medio Ambiente y Desarrollo Sostenible. Docente de la Universidad Nacional Hermilio Valdizan de Huánuco Huánuco, Perú.

${ }^{2}$ Lic. Enfermería. Dr. Salud Pública. Docente de la Universidad Nacional Hermilio Valdizan de Huánuco - Huánuco, Perú.

3 Médico Internista. Dr. en Medicina. Docente de la Universidad Nacional Hermilio Valdizan de Huánuco - Huánuco, Perú.
} 


\section{INTRODUCCIÓN}

Son muchas las enfermedades e infecciones compartidas naturalmente entre los animales vertebrados y el hombre según el Instituto Colombiano Agropecuario, la Organización Panamericana de la Salud y la Organización Mundial de la Salud $(1,2)$. Casi todos los episodios de enfermedades infecciosas durante los últimos 10 años han incluido los agentes infecciosos zoonóticos (1). Por esta razón, se ha declarado la importancia de las zoonosis en la aparición de las infecciones humanas, lo cual no puede subestimarse(3).

Dentro de una amplia gama de enfermedades zoonóticas, las parasitarias, tienen poca importancia dentro del contexto de la Salud Pública. La mayoría de ellas no dan lugar a emergencias epidemiológicas notables, y no están sujetas a notificación obligatoria en la mayoría de los países.

La mascota infectada constituye un factor de riesgo para la población en contacto especialmente los niños, adultos mayores y personas inmunocomprometidas (4).

Los cánidos son hospedadores de diversos parásitos, de los cuales los más comunes y ampliamente diseminados son: nematodos gastroentéricos, cestodos y protozoarios (5).

La ingestión de quistes y ooquistes, así como la penetración a través de la piel por larvas infectantes, constituye la ruta de entrada para los parásitos que producen enfermedades en el hombre, principalmente las zoonosis, según el Institute of Medicine (6).

Los resultados obtenidos en estudios similares sobre prevalencia de estas parasitosis en Latinoamérica son muy variables. En el Perú, la mayoría de estudios se han realizado en Lima, siendo escasos los realizados en otros departamentos. Los primeros estudios realizados fueron sobre contaminación de parques públicos con huevos de Toxocara spp., entre ellos se encuentran los estudios realizados por Guerrero (1975) en parques públicos de Lima Metropolitana dando un resultado de $24 \%$ (7), estudios posteriores señalaron niveles similares de contaminación.
En Ica, existe un estudio sobre la prevalencia de helmintos enteroparásitos zoonóticos y factores asociados en Canis familiaris en una zona urbana de esta ciudad (8).

En la Provincia de Huánuco existen escasos estudios sobre contaminación por parásitos zoonóticos, entre ellos el que se realizó en el 2001 sobre la contaminación de los parques públicos con huevos de Toxocara sp. en la Ciudad de Huánuco, donde se encontró que de 12 parques públicos muestreados los cuales están ubicados en el área urbana de la Ciudad de Huánuco, 9 de ellos resultaron estar contaminados con huevos de Toxocara sp, hallando una prevalencia de $75 \%$ (9). Desde esa fecha no se ha dado importancia al estudió sobre esta parasitosis zoonótica, por lo tanto se desconocía el grado de infección de estas parasitosis.

Comparado a los estudios que se hizo anteriormente en esta Provincia; en la presente investigación se estudió la prevalencia de cinco de los parásitos más zoonóticos más importantes (Toxocara canis, Toxascaris leonina, Ancylostoma caninum, Dipylidium caninum y Taenia sp.), que como ya se mencionó son un gran problema en la salud pública y también en salud ambiental.

Objetivo general: Determinar la relación de la prevalencia de enteroparásitos zoonóticos en perros (Canis familiaris) y el nivel de cultura ambiental orientado a mascotas en el Centro Poblado La Esperanza, Huánuco.

Hipótesis: Existe relación entre la prevalencia de enteroparásitos zoonóticos en perros (Canis familiaris) y el Nivel de cultura ambiental orientado a mascotas en el Centro Poblado La Esperanza, Huánuco.

\section{MATERIAL Y MÉTODOS}

El presente estudio es una investigación aplicada, pertenece al diseño no experimental, se enmarcó en el nivel descriptivo, explicativo y correlacional.

Diseño epidemiológico: transversal o de prevalencia. 


\section{Dónde:}

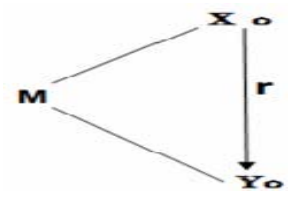

$\mathbf{M}=$ Representa a la muestra en estudio: Heces de perros de La Esperanza.

Xo $=$ Representa la variable independiente: Nivel de cultura ambiental orientado a mascotas, en los pobladores.

Yo $=$ Representa la variable dependiente: Prevalencia de Enteroparásitos zoonóticos en perros.

$\mathbf{r}=$ Representa la relación de las variables en estudio.

Población: Estuvo comprendido por personas que tenían uno o más perros en casa, en el Centro Poblado La Esperanza, que pertenece al Distrito de Amarilis, Provincia de Huánuco, donde se estimó una población canina de 391 perros para el año 2014.

Muestra: Se utilizó la fórmula para estudios transversales en población finita conocida. La selección de la muestra fue por el método probabilístico por la modalidad de muestreo sistemático. Para efectos del estudio se consideró un nivel de confianza de $95 \%$, una estimación de $5 \%$ y una prevalencia de $10 \%$ que se obtuvo al promediar los cinco parásitos en un estudio de prevalencia de helmintos enteroparásitos zoonóticos y factores asociados en Canis familiaris en una zona urbana de la ciudad de Ica, Perú (8).

Datos:

$$
\begin{aligned}
& \mathrm{N}=391 \\
& \mathrm{e}=5 \%=0,05 \\
& \text { Prevalencia de }=10 \%=0,1 \\
& \mathrm{Z}=1,96 \text { (tabla de distribución normal para el } \\
& 95 \% \text { de confiabilidad y } 5 \% \text { error) } \\
& \mathrm{p}=0,1 \\
& \mathrm{q}=1-\mathrm{p}
\end{aligned}
$$

Se calculó haciendo uso de la siguiente fórmula:

$$
\begin{aligned}
& \mathrm{n}=\frac{(1,96) 2 \quad(0,1) \quad(1-0,1) \quad(391)}{(391)(0,05) 2+(1,96) 2(0,1)(1-0,1)} \\
& \mathrm{n}=102,16 \rightarrow(\text { redondeando a 103). }
\end{aligned}
$$

Para fines prácticos del estudio se aumentó la muestra a 104 perros.

Se consideró en el estudio a perros con dueños mayores de 2 meses de edad, de ambos sexos, de todas las razas que habitan en La Esperanza y que no hayan recibido tratamiento antihelmíntico en los últimos 15 días antes de la toma de muestra. Los animales se seleccionaron mediante un muestreo bietápico, para seleccionar la vivienda (unidad primaria de muestreo) se utilizó el muestreo sistemático. Si el propietario tenía más de un perro, entonces para seleccionar el animal en la vivienda (unidad secundaria de muestreo) se utilizó un muestreo aleatorio, para lo cual se realizó un sorteo de los perros que viven allí. En todos los casos se solicitó la autorización de los dueños para incluir sus mascotas en el estudio. Cuando los dueños no aceptaron que sus animales ingresen al estudio, fue reemplazado por otra vivienda más cercana.

\section{Análisis e interpretación de los datos}

Análisis descriptivo: Para cada una de las variables se tuvo en cuenta las medidas de tendencia central y dispersión para las variables cuantitativas y de porcentajes para las variables categóricas.

Análisis inferencial: Se realizó el análisis bivariado mediante la Prueba de Spearman para Correlacionar la prevalencia de enteroparásitos zoonóticos en perros y el nivel de cultura ambiental orientado a mascotas. Para el procesamiento de los datos se utilizó el paquete estadístico SPSS versión 21,0.

\section{Definición operativa del instrumento de recolección de datos}

Cuestionario: Es un Instrumento elaborado con un grupo de preguntas abiertas y cerradas; fue validado por juicio de 05 expertos con dominio del tema a investigar, el coeficiente de validación: Muy buena (por estar en: 61 a 80\%), de acuerdo al puntaje obtenido el instrumento fue aplicable; dicho instrumento se enmarcó en base a las siguientes dimensiones:

a) Ficha para conocer los datos del poblador y de su perro.

b) Encuesta para determinar el nivel de cultura ambiental de los pobladores, el conocimiento sobre las enfermedades parasitarias zoonóticas y el conocimiento del manejo adecuado de excretas de perros por parte de los pobladores. Este cuestionario estuvo dirigido a los pobladores, con la finalidad de recopilar toda la información necesaria para determinar el nivel de cultura ambiental orientado a mascotas en este estudio.

c) Ficha de análisis coproparasitológico para la detección de enteroparásitos zoonóticos de los perros en estudio.

d) Ficha de registro general de los análisis de heces de todos los perros en estudio,

Prueba Coproparasitológico para diagnóstico de helmintos: Se evaluaron dos muestras por animal mediante el Método de Graban y de concentración (Método de flotación de Sheather y Método de 
INVESTIGACIÓN ORIGINAL ORIGINAL RESEARCH
Prevalencia de enteroparásitos zoonóticos en perros (canis familiaris) y el nivel de cultura ambiental orientado a mascotas en Huánuco Huerto E, Fonseca A y Dámaso B. sedimentación simple en copas). El método para detectar la presencia de los parásitos en estudio fue el Método de flotación fecal, el cual se utilizan para separar los parásitos en todos sus estadios de otros objetos, basados en sus diferentes densidades. Se empleó la solución sacarosa. Esta solución se recomienda para el diagnóstico de helmintos.

\section{RESULTADOS}

La prevalencia total por uno o más helmintos enteroparásitos zoonóticos en perros del Centro
Poblado La Esperanza, fue alta, 92,3\%. Para Ancylostoma caninum (72,1\%), para Toxocara canis $(54,8 \%)$, para Taenia sp. $(20,2 \%)$, para Toxascaris leonina $(19,2 \%)$ y para Dipylidium caninum (13,5\%). Según el nivel de cultura ambiental orientado a mascotas, de los 104 dueños encuestados, 8 de ellos (7,7\%) posee el nivel bajo, 36 de ellos $(34,6 \%)$ poseen el nivel medio y 60 de ellos $(57,7 \%)$ poseen el nivel alto. No existe relación significativa, ya que se obtuvo un valor rho $=-0,044$, con un $\mathrm{P}$ (sig.) $=0,658$, entre la prevalencia de enteroparásitos zoonóticos en perros y el nivel de cultura ambiental orientado a mascotas.

Tabla 1: Prevalencia total por uno o más helmintos enteroparásitos zoonóticos en Canis familiaris, la Esperanza - Huánuco, 2014.

\begin{tabular}{lcc}
\hline Resultado & Frecuencia & \% \\
\hline Positivo & 96 & 92,3 \\
Negativo & 8 & 7,7 \\
Total & $\mathbf{1 0 4}$ & $\mathbf{1 0 0 , 0}$ \\
\hline
\end{tabular}

Tabla 2: Prevalencia por helmintos enteroparásitos zoonóticos en Canis familiaris, la Esperanza - Huánuco, 2014.

\begin{tabular}{lcc}
\hline Especies & Positivos & Prevalencia (\%) \\
\hline Ancylostoma caninum & 75 & 72,1 \\
Toxocara canis & 57 & 54,8 \\
Taenia sp. & 21 & 20,2 \\
Toxascaris leonina & 20 & 19,2 \\
Dipylidium caninum & 14 & 13,5 \\
\hline
\end{tabular}

Tabla 3: Distribución según Nivel de Cultura Ambiental Orientado a mascotas; de los dueños de los canes, la Esperanza - Huánuco, 2014.

\begin{tabular}{lcc}
\hline $\begin{array}{l}\text { Nivel de cultura ambiental orientado a } \\
\text { mascotas }\end{array}$ & Frecuencia & \% \\
& & \\
\hline Nivel Bajo & 8 & 7,7 \\
Nivel Medio & 36 & 34,6 \\
Nivel Alto & 60 & 57,7 \\
Total & $\mathbf{1 0 4}$ & $\mathbf{1 0 0}$ \\
\hline
\end{tabular}


INVESTIGACIÓN ORIGINAL ORIGINAL RESEARCH
Prevalencia de enteroparásitos zoonóticos en perros (canis familiaris) y el nivel de cultura ambiental orientado a mascotas en Huánuco

Tabla 4: Comparación entre la Prevalencia de enteroparásitos zoonóticos en perros y el Nivel de Cultura Ambiental orientado a mascotas, la Esperanza - Huánuco, 2014.

\begin{tabular}{cccc}
\hline \multirow{2}{*}{ Nivel de Cultura Ambiental } & \multicolumn{2}{c}{ Prevalencia } \\
\cline { 2 - 4 } & & Positivo & Negativo \\
\hline \multirow{2}{*}{ Bajo } & $\mathrm{N}$ & 8 & 0 \\
& $\%$ & 8,3 & 0,0 \\
\multirow{2}{*}{ Medio } & $\mathrm{N}$ & 33 & 3 \\
& $\%$ & 34,4 & 37,5 \\
\multirow{2}{*}{ Alto } & $\mathrm{N}$ & 55 & 5 \\
& $\%$ & 57,3 & 62,5 \\
\multirow{2}{*}{ Total } & $\mathbf{N}$ & $\mathbf{9 6}$ & $\mathbf{8}$ \\
& $\mathbf{\%}$ & $\mathbf{1 0 0 , 0}$ & $\mathbf{1 0 0 , 0}$ \\
\hline
\end{tabular}

Tabla 5: Prueba de Spearman para Correlacionar la Prevalencia y Nivel de Cultura Ambiental orientado a mascotas.

\begin{tabular}{|c|c|c|c|}
\hline $\begin{array}{l}\text { Correlación de } \\
\text { Spearman }\end{array}$ & & Prevalencia & $\begin{array}{c}\text { Nivel de Cultura } \\
\text { Ambiental }\end{array}$ \\
\hline \multirow{3}{*}{ Prevalencia } & $\begin{array}{l}\text { Coeficiente de } \\
\text { correlación }\end{array}$ & 1 & $-0,044$ \\
\hline & Sig. (bilateral) & & 0,658 \\
\hline & $\mathrm{N}$ & 104 & 104 \\
\hline \multirow{3}{*}{$\begin{array}{l}\text { Nivel de Cultura } \\
\text { Ambiental }\end{array}$} & $\begin{array}{l}\text { Coeficiente de } \\
\text { correlación }\end{array}$ & $-0,044$ & 1 \\
\hline & Sig. (bilateral) & 0,658 & e \\
\hline & $\mathrm{N}$ & 104 & 104 \\
\hline
\end{tabular}

\section{DISCUSIÓN}

La prevalencia total por uno o más helmintos enteroparásitos zoonóticos en perros fue de $92,3 \%$; resultado superior al hallado en la ciudad de Ica, en un estudio de "Prevalencia de helmintos enteroparásitos zoonóticos y factores asociados en perros", donde se obtuvo una prevalencia por uno o más helmintos enteroparásitos de 40,12\% (A. caninum, $T$. canis, Taenia sp., T. leonina y D. caninum $)^{(\mathbf{8})}$. Del modo similar fue muy superioir en otro estudio de "Prevalencia de helmintos gastrointestinales en caninos de la ciudad de Cuenca en Ecuador"; donde se obtuvo una prevalencia de $15,45 \%$ (A. caninum, T. canis, Uncinaria stenocephala, Trichuris vulpis, Taenia spp. y D. caninum) (10). La superioridad del resultado de nuestro estudio comparado con la ciudad de Ica, se debe probablemente a que sus condiciones sucioculturales $\mathrm{y}$ medioambientales son diferentes a la ciudad de Huánuco, sobre todo las condiciones medioambientales donde se encuentren los parásitos determinará el tiempo de duración de su ciclo de vida. Caso similar sucede en la ciudad de Cuenca, Ecuador, donde se obtuvo una prevalencia bastante menor comparada al presente estudio, ya que en esta ciudad se vienen aplicando programas educativos sobre bienestar animal y tenencia responsable de mascotas en escolares (11), que a su vez estos escolares transmiten los conocimientos aprendidos a los demás integrantes de su familia y también la diferencia de la prevalencia se deba a factores medio ambientales distintos a la ciudad de Huánuco. En la diferencia en los resultados, a su vez influyeron los factores socioculturales y los diferentes estudios se realizaron en diferentes épocas del año y se usaron diferentes pruebas coproparasitológicas. Los resultados obtenidos en el presente estudio indican que la zona de La Esperanza debe ser considerada como de riesgo potencial para adquirir parásitos zoonóticos en humanos. Es posible que estudios similares en otras zonas del país, entre ellos en Huánuco, indiquen resultados similares. Por lo tanto se recomienda desparasitar periodicamentente a los perros en estas zonas del país por su alto potencial zoonótico y también porque alteran la 
salud de los mismos perros produciendo infecciones intensas particularmente en cachorros, pueden causar anorexia, decaimiento, pobre ganancia de peso, deshidratación, deficiencias de hierro, anemia severa (hipocrómica microcítica), pérdida intestinal de sangre, melena y muerte debido a su voraz hábito de succionar sangre; gran número de larvas en cachorros puede causar neumonía durante su migración pulmonar, por mencionar solo al Ancylostoma caninum (12).

Según el nivel de cultura ambiental orientado a mascotas, no se halló estudios similares para poder comparar nuestro estudio, pero luego de encontrar en los resultados; que de los 104 dueños encuestados, 8 de ellos $(7,7 \%)$ posee el nivel bajo, 36 de ellos (34,6\%) poseen el nivel medio y 60 de ellos $(57,7 \%)$ poseen el nivel alto, podríamos observar que la mayoría posee nivel alto, seguido del nivel medio y en menor porcentaje el nivel bajo. Probablemente esto se debe a que las personas en la actualidad acceden a diversos medios de comunicación, entre ellos la televisión, la radio, el internet; dichos medios proporcionan información respecto a temas medio ambientales orientados a la ecología y desarrollo sostenible, problemática actual y medidas preventivas en cuanto al cuidado del medio ambiente; esto se observa casi todos los días en los distintos medios de comunicación informándonos sobre: cambio climático, calentamiento global, destrucción de la capa de ozono, destrucción de los bosques y selvas tropicales, contaminación de los océanos, contaminación atmosférica, pérdida de diversidad biológica debido a la deforestación, contaminación de suelos, entre otros temas más; también se mencionan temas sobre Tenencia Responsable de Mascotas, donde abarcan temas de bienestar animal, zoonosis y contaminación por excremento de mascotas. Es por todo esto que en nuestros resultados la mayoría de los encuestados pertenecen a un nivel de cultura ambiental orientado a mascotas medio y alto.

Finalmente al evaluar la relación entre la prevalencia de enteroparásitos zoonóticos en perros y el nivel de cultura ambiental orientado a mascotas, observamos que no existe relación significativa ya que se obtuvo un valor rho = 0,044 , con un $\mathrm{P}$ (sig.) $=0,658$. No se halló estudios similares para poder comparar nuestros resultados. Pero luego de encontrar este resultado, podemos observar aparentemente de que si los encuestados presentan en su mayoría un alto nivel de cultura ambiental, ¿como se explica que la prevalencia de enteroparásitos zoonóticos resulto alta (92,3\%)?; la explicación sería la siguiente: Si bien es cierto los dueños de los perros en estudio, tienen un nivel alto de cultura ambiental, por lo mencionado anteriormente ya que los medios de comunicación mantienen actualizados sus conocimientos en temas medio ambientales y tenencia responsable de mascotas, pero a su vez estos dueños no están poniendo en práctica lo aprendido. Esto se confirma en la pregunta que se les hizo cuando se realizó la encuesta en cuanto a la frecuencia de desparasitación de sus perros, 58 de ellos $(55,8 \%)$ nunca fueron desparasitados y 21 de ellos $(20,2 \%)$ fueron desparasitados una sola vez en su vida, entonces solo 46 (44,2\%) canes fueron desparasitados en algún momento de su vida, considerándose una desparasitación ineficaz. Esta sería la explicación al no encontrar una relación estadística significativa entre ambas variables.

Por lo tanto entendemos que a pesar que los dueños conocen sobre la desparasitación de los perros, estos dueños no estarían siendo responsables en cuanto al control de la desparasitación de sus perros, pueden conocer pero aún no han sido concientizados, problema que debería ser solucionado con Educación en Tenencia Responsable de Mascotas y Bienestar Animal.

\section{AGRADECIMIENTOS}

A los pobladores del Centro Poblado La Esperanza que participaron en el presente estudio.

\section{AUTOR DE CORRESPONDENCIA}

Edward Huerto-Medina

Universidad Nacional Hermilio Valdizan de

Huanuco, Distrito de Pillco Marca

Av. Universitaria 601-607-Cayhuayna

Huánuco - Perú.

Teléfono: +51-(062) 591060 - Anexo: 0103.

E-mail: edwardhm_10@hotmail.com

\section{REFERENCIAS BIBLIOGRÁFICAS}

1. Instituto Colombiano Agropecuario y Organización Panamericana de la Salud. Salud Pública Veterinaria, protección sanitaria, y desarrollo agropecuario. Simposio Internacional. Memorias; Bogotá, Colombia; 11 de junio 2002, pp. 83-87.

2. Organización Panamericana de la Salud/Organización Mundial de la Salud. 14 
Reunión Interamericana a Nivel Ministerial en Salud y Agricultura. RIMSA. Las enfermedades desatendidas en las poblaciones postergadas, con énfasis en las zoonosis. Ciudad de México; 18 abril 2005.

3. Ambroise-Thomas, P. Emerging parasite zoonoses: the role of host-parasite relationship. International Journal of Parasitology. 2000; 30: 1361-1367.

4. Robertson, I. D. The role of companion animals in the emergence of parasitic zoonoses. International Journal for Parasitology. 2000; 30: 1369-1377.

5. Martínez, B. T., Gutiérrez, C. E. M., Alpízar, S. E. y Pimienta, L. R. Contaminación parasitaria en heces de perros, recolectadas en calles de la ciudad de San Cristóbal de Las Casas, Chiapas, México. Vet. Mex. 2008; 39(2): 173-180.

6. Institute of Medicine (IOM): The Emergent of Zoonotic Diseases - Understanding the impact Animal and Human Health. Washington, DC National Academy Press. 2002.

7. Guerrero, M. O. Estudio de la contaminación de parques públicos de Lima Metropolitana con huevos de Toxocara spp. Tesis de Bachiller. Facultad de Medicina Veterinaria, Univ. Nacional Mayor de San Marcos. 1975; Lima, p.12.

8. Trillo, M. del P., Carrasco, A. J. y Cabrera, R. Prevalencia de helmintos enteroparásitos zoonóticos y factores asociados en Canis familiaris en una zona urbana de la ciudad de
Ica, Perú. Parasitol Latinoam. 2003; 58: 136 141, FLAP.

9. Huaraca, F. Contaminación de parques públicos con huevos de Toxocara sp. en el Distrito de Huánuco (Tesis para obtener el título de Médico Veterinario. Facultad de Medicina Veterinaria y Zootecnia de la Universidad Nacional Hermilio Valdizán. 2001; Huánuco - Perú.

10. Ramón, G. F. "Prevalencia de Helmintos Gastrointestinales (Céstodos y Nematodos) en caninos de la ciudad de Cuenca". Tesis de Grado previa a la obtención del Título de Médico Veterinario Zootecnista. Universidad de Cuenca Facultad de Ciencias Agropecuarias Escuela de Medicina Veterinaria y Zootecnia. 2012.

11. Cueva, M. "Evaluación del Impacto de un programa educativo sobre Bienestar Animal y Tenencia Responsable de Mascotas en escolares de las unidades educativas mixtas del Cantón Cuenca. Tesis previa a la obtención del título de Magíster en Investigación de la Salud. Universidad de Cuenca Facultad de Ciencias Médicas. 2011; p. 1.

12. Dvorak, G., Rovid-Spickler, A. y Roth, J. Editores. Handbook for zoonotic diseases of companion an imals. (1a ed.). Ames: The Center Food Security and Public Health. 2008; pp. 138- 41.

Recibido: $30 / 09 / 2015$

Aceptado: $20 / 12 / 2015$ 